EXTENDED REPORT

\title{
Macular function and morphology after peeling of idiopathic epiretinal membrane with and without the assistance of indocyanine green
}

\author{
J Hillenkamp, P Saikia, F Gora, H G Sachs, C P Lohmann, J Roider, W Bäumler, V-P Gabel
}

Br J Ophthalmol 2005;89:437-443. doi: 10.1136/bjo.2004.051250

See end of article for authors' affiliations

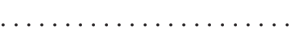

Correspondence to: Dr Jost Hillenkamp, Eye Hospital, University of Regensburg, Franz-Josef-

Strauss-Allee 11, D-93042

Regensburg, Germany; hillenka@hotmail.com

Accepted for publication 1 August 2004
Aim: To investigate macular function and morphology after surgical removal of idiopathic epiretinal membrane (IEM) with and without assistance of indocyanine green (ICG).

Methods: A retrospective study as a consecutive case series, of 39 patients with IEM. 39 patients, 23 female, 16 male, mean age 67 years, underwent standard three port pars plana vitrectomy with removal of epiretinal membrane. Two groups of patients were consecutively operated: in 20 patients ICG $0.1 \%$ in glucose $5 \%$ was used to stain the epiretinal membrane. 19 patients underwent the identical procedure but without use of ICG. Postoperative follow up was 1-92 months (mean 15.5 months). Functional outcome was assessed with subjective improvement, best corrected visual acuity (BCVA), Amsler grid test, $10^{\circ}$ and $30^{\circ}$ automated perimetry (Heidelberg visual field analyser) (HFA), and Goldmann kinetic perimetry. Macular morphology was assessed with stereoscopic biomicroscopy and optical coherence tomography (OCT). The main outcome measures were macular function as determined by BCVA, presence of visual field defects, and metamorphopsia as determined by Amsler grid test, macular morphology as determined by slit lamp biomicroscopy, and OCT.

Results: BCVA improved in 28 patients, remained unchanged in eight patients, and decreased in three patients. Improvement of BCVA was statistically significant in both groups $(p=0.003)$. Mean BCVA in patients operated with ICG improved from 0.33 preoperatively to 0.53 postoperatively. Mean BCVA in patients operated without ICG improved from 0.32 preoperatively to 0.54 postoperatively. Reduction of macular oedema as measured by OCT was statistically significant in both groups $(p<0.01)$. There was no statistically significant difference in postoperative BCVA, macular oedema as measured by OCT, postoperative Amsler grid test, and subjective improvement between the two groups. The incidence of residual or recurrent epiretinal membrane was greater in the group operated without ICG $(p=0.014)$. Visual field defects were detected in one patient operated with ICG and in three patients operated without ICG.

Conclusions: Removal of epiretinal tissue with or without assistance of ICG improved visual function and reduced macular oedema in most patients. Adverse effects clearly attributable to the use of ICG were not observed but further investigation is warranted. diopathic epiretinal membrane (IEM) is characterised by the formation of epimacular membraneous tissue without any known underlying retinal disease. The prevalence of this entity is $2 \%$ in those under the age of 60 years and $12 \%$ beyond the age of 70 years. ${ }^{1}$ IEM is associated with macular dysfunction probably related to an impairment of the inner retinal layers, ${ }^{2}$ which is similar to that found in aphakic cystoid macular oedema. ${ }^{3}$ In fact, optical coherence tomography (OCT) studies have demonstrated an increased retinal thickness of the macula, ${ }^{4-6}$ which decreased significantly after surgical membrane peeling but remained greater than the macular retinal thickness of the normal fellow eyes. ${ }^{7}$

Recently, indocyanine green (ICG) has been introduced to stain and visualise epiretinal membranes and the internal limiting membrane to facilitate the delicate surgical manoeuvre of their removal in epiretinal membrane ${ }^{8-10}$ and macular hole surgery. ${ }^{1-13}$ In the surgical treatment of IEM the delamination of epiretinal membranes usually leads to a complete removal of all epiretinal tissue and peeling of the underlying internal limiting membrane is only attempted when complete removal of epiretinal membranes cannot be readily achieved. However, histological studies have shown that the internal limiting membrane is often removed together with fibrocellular epiretinal membranes even when it was not intended. ${ }^{14} 15$

Several authors have reported good functional outcome of ICG assisted vitrectomy, ${ }^{12}{ }^{16-18}$ whereas some authors have reported less favourable results in visual acuity, ${ }^{819} 20$ and significant visual field defects when intraocular ICG was used. ${ }^{8}{ }^{19-21}$ Because of this controversy, a possible toxic effect of ICG on the inner retina has become the subject of an ongoing debate.

It was the aim of this study to retrospectively compare the functional and morphological outcome of epiretinal membrane peeling as a treatment for IEM with and without the assistance of ICG.

\section{PATIENTS AND METHODS}

The present study was designed as a retrospective analysis of two consecutive interventional case series. A total of 39 patients (23 female, 16 male) with a mean age of 67 years with IEM were retrospectively recruited. All patients

Abbreviations: BCVA, best corrected visual acuity; HFA, Heidelberg visual field analyser; ICG, indocyanine green; IEM, idiopathic epiretinal membrane; OCT, optical coherence tomography 
complained preoperatively of reduced visual acuity and metamorphopsia. Twenty patients were operated with the aid of ICG and 19 patients underwent the same surgical procedure but without the aid of ICG. All patients of the group operated without ICG were operated before the introduction of ICG staining in macular surgery in our hospital during 2001. All patients were operated by experienced surgeons (HGS, CPL, JR, V-PG). Surgeons commenced applying ICG at different times during 2001. Patients with other retinal disease, glaucoma or postoperative retinal detachment were excluded from the study.

\section{Surgical technique}

All patients underwent standard three port pars plana vitrectomy, including where necessary the induction of a posterior vitreous detachment. Balanced salt solution (BSS; Alcon Laboratories, Fort Worth, TX, USA) was used as an irrigation solution. In the group operated without ICG the epiretinal membrane was engaged with intraocular forceps to create a flap and then peeled from the nerve fibre layer usually in a capsulorrhexis fashion ${ }^{22}$ before a routine closure without the use of tamponade agents. In the group operated with ICG the continuous BSS irrigation was briefly stopped and less than $0.5 \mathrm{ml}$ of ICG (Pulsion Medical Systems AG, Munich, Germany) at a $0.1 \%$ concentration dissolved in glucose $5 \%$ was injected into the BSS filled globe just above the posterior pole. The osmolarity of this solution was 296 mOsm as measured with an osmometer (Knauer, Berlin, Germany). After less than 1 minute, the irrigation was restarted and thus the dye was quickly washed out of the globe. The epiretinal membrane was stained at a faint green colour and was clearly visible under illumination from the standard light pipe of the vitrectomy instrument. The stained membrane was engaged with intraocular forceps and peeled in the same manner as in the patients operated without ICG before routine closure without the use of tamponade agents. Complete removal of all epiretinal membranous tissue was attempted in all patients in both groups. The delamination of epiretinal membranous tissue usually led to a complete removal of all visible epiretinal tissue and peeling of the underlying internal limiting membrane was only attempted when complete removal of epiretinal membranes could not be readily achieved. In some of these cases, the surgeon injected ICG a second time to clearly stain the underlying ILM. In both groups some patients underwent standard phacoemulsification intraocular lens surgery, either concomitant or as a second operation after the membrane peeling. Preoperative and postoperative lens status (phakic/pseudophakic) was not statistically significantly different between the two groups (preoperative status: $\mathrm{p}=0.4$ and postoperative status: $p=0.7, \chi^{2}$ test, Fisher's test) (table 1).

\section{Clinical assessment of function and morphology}

Patients were examined at 1-92 months (mean 15.5 (SD 17.5) months) after surgical membrane peeling. Subjective improvement of overall visual function and subjective improvement of metamorphopsia was recorded. Functional outcome was assessed with best corrected decimal visual acuity with a Snellen chart (BCVA), Amsler grid test, $10^{\circ}$ and $30^{\circ}$ automated perimetry (Heidelberg visual field analyser, HFA), and Goldmann kinetic perimetry. The incidence of residual epiretinal membrane was assessed with stereoscopic biomicroscopy. In most patients, macular oedema was assessed by OCT measurement of the macular retinal thickness (OCT 1, Zeiss-Humphrey and OCT 3, ZeissMeditec, Jena, Germany).

The results were statistically analysed using SPSS 10.0 software (SPSS Inc, Chicago, IL, USA) and Microsoft-Excel (Microsoft Corp, Redmond, WA, USA).

\section{Measurement of the emission spectrum of the light} source

The spectral distribution of the light source emission was measured using an optical multichannel analyser (Spex 270M, Jobin Yvon, Munich, Germany). The emission of the light source was fed into the spectrograph $(0.1 \mathrm{~mm}$ entrance slot) and detected by the cooled CCD camera in the spectral range of 590-908 $\mathrm{nm}$ with a resolution of $1 \mathrm{~nm}$. The optical output of the light source was measured through the light fibre. The fibre of the light source was directly connected to the light fibre of the spectrograph to measure qualitatively the light distribution.

\section{RESULTS}

We investigated the outcome of surgical peeling of IEM in two groups of patients: patients operated with and without the aid of ICG.

The following parameters were not statistically significantly different between the two groups: 15 of 20 patients operated with ICG reported an overall subjective improvement of visual function since the operation versus 16 of 19 patients in the group operated without ICG $\left(p=0.7, \chi^{2}\right.$ test, Fisher's test). Metamorphopsia had subjectively improved in 15 of 20 patients operated with ICG and in 13 of 19 patients operated without ICG $\left(p=0.73, \chi^{2}\right.$ test, Fisher's test $)$.

One patient in the group operated with ICG had a paracentral nasal visual field defect in the HFA and Goldmann perimetry (fig 1, patient 15). Three patients in the group operated without ICG had visual field defects: One lower temporal peripheral defect in the Goldmann perimetry (fig 2, patient 23), one paracentral nasal defect in the HFA and Goldmann perimetry (fig 3, patient 24), and one paracentral defect in the HFA $10^{\circ}$ perimetry (fig 4, patient 30). There were two additional patients with visual field defects in each group but these non-specific defects were recorded only by HFA with extensive losses of fixation suggesting an unreliable test result which was not reproducable by Goldmann perimetry (table 1). These defects were not taken into account in the data analysis. All visual field defects were asymptomatic.

The normalised emission spectrum of the light source and the normalised absorption spectra of ICG dissolved in water or in $5 \%$ glucose showed an overlap (fig 5 ).

The analysis of all other parameters is represented in tables $1-4$.

\section{DISCUSSION}

Our results show no statistically significant difference in macular function as assessed by preoperative and postoperative BCVA, Amsler grid test, or subjective improvement of overall visual function and metamorphopsia between the groups operated with and without the aid of ICG. BCVA improved in both groups. In accordance with the findings of others ${ }^{7}$ macular oedema improved with surgery. The reduction of macular oedema as assessed by OCT measurement of foveal retinal thickness was not statistically significantly different between the two groups. The incidence of residual or recurrent epiretinal membrane was greater in the group operated without ICG. This finding may be related to the longer follow up period which could be a reason for a greater number of recurrent membrane formation in that group. Alternatively, this finding may be related to a greater number of incomplete removals of epiretinal membrane possibly because not all membranous tissue could be intraoperatively visualised without the aid of ICG staining. A distinction between these two possible causes must be made by a future suitably designed prospective randomised trial. Furthermore, the present study has some of the usual limitations of retrospective analyses. We cannot exclude the presence of the 


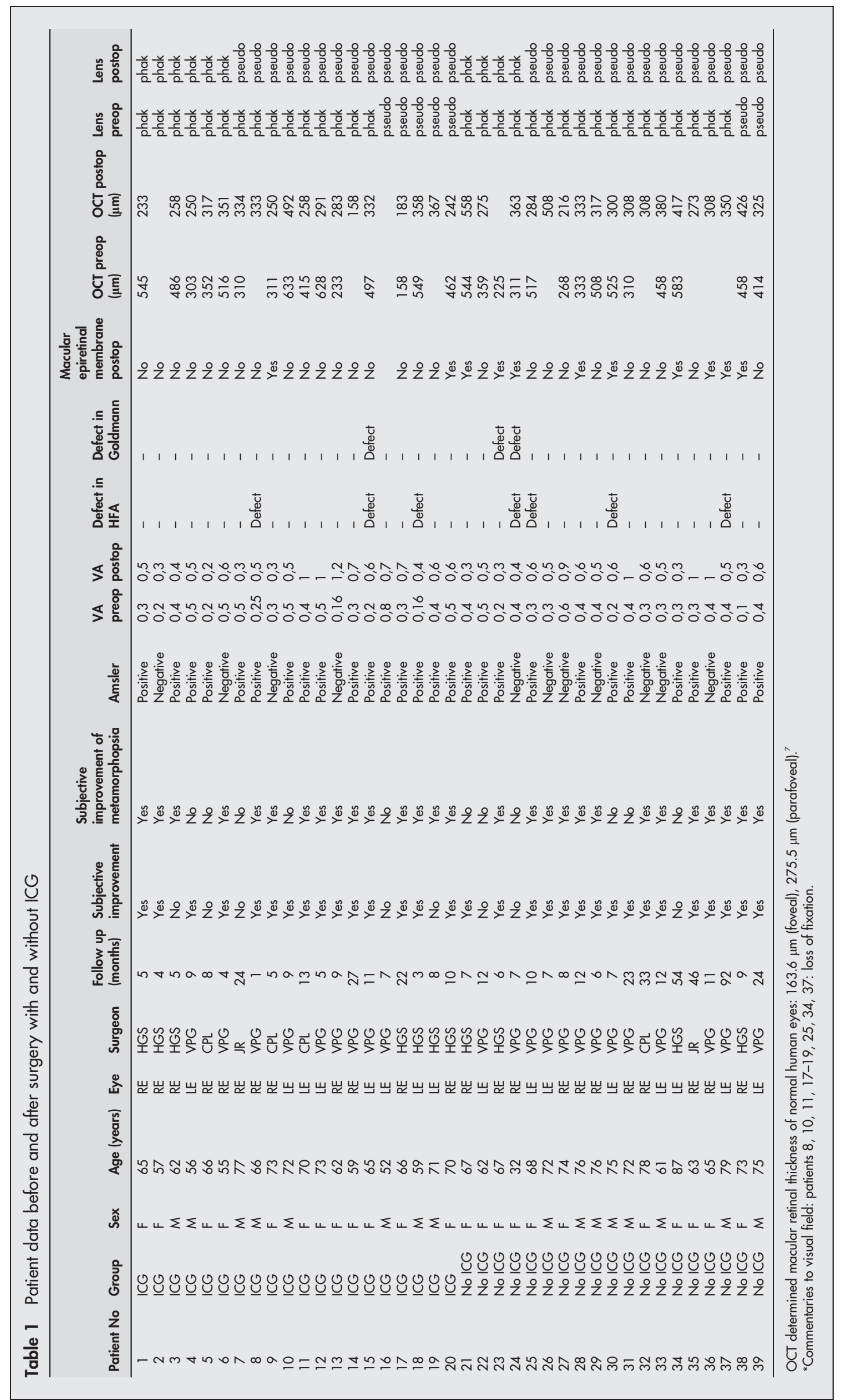




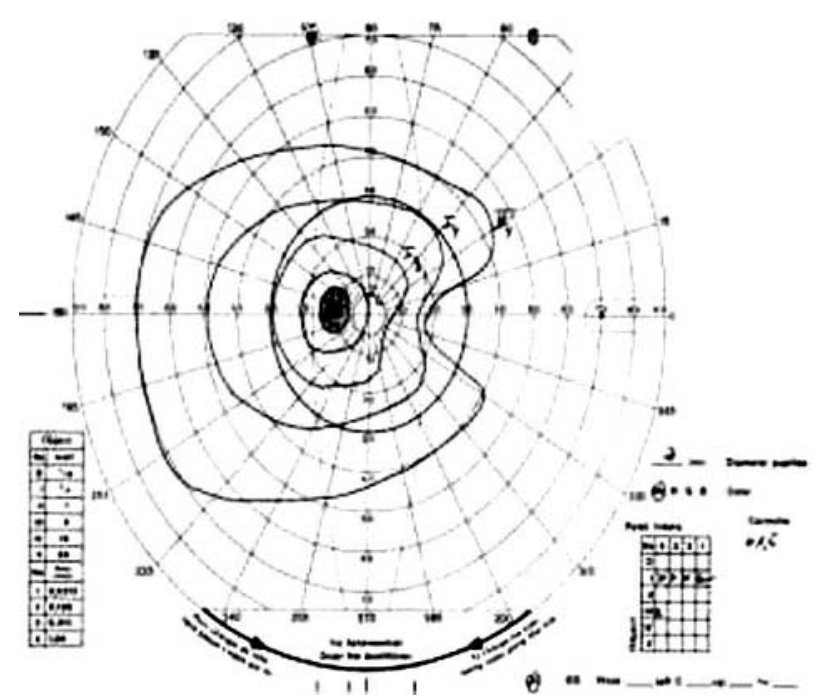

Figure 1 Patient 15. Operated with ICG. Paracentral nasal defect.

observed visual field defects before surgery. The relatively large number of surgeons and the fact that examiners were not blinded are possible sources of confounding. We cannot exclude that comparing non-contemporary groups of patients is a source of confounding as the surgeons may have had improved their skills when operating the second group of patients. The patients operated with ICG were younger than the patients operated without ICG. However, since all patients except one 32 year old patient were in the age group above 50 years the difference of patient ages is unlikely to have had a significant impact on the investigated outcome measures.

Our findings in postoperative visual acuity are in accordance with other authors who described a positive functional outcome of ICG assisted vitreous surgery. ${ }^{12}{ }^{16-18}$ Our findings are in contrast with authors who described less favourable postoperative visual acuity ${ }^{819} 20$ and visual field defects ${ }^{819-21}$ after ICG assisted vitreous surgery. These reports have contributed to the concern that ICG as used in macular surgery may be toxic to the inner retina.

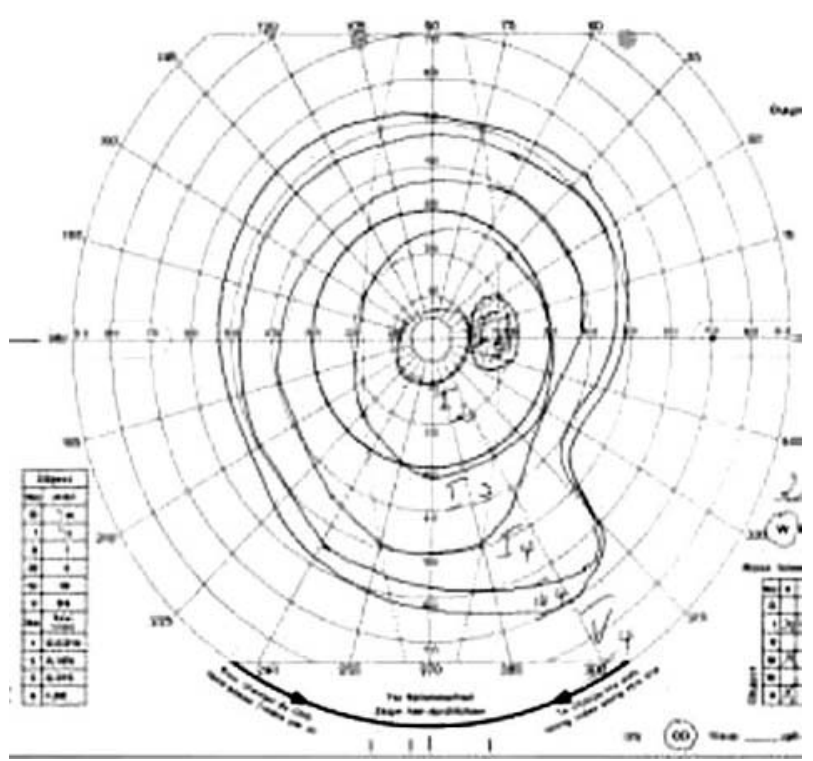

Figure 2 Patient 23. Operated without ICG. Peripheral lower temporal defect.

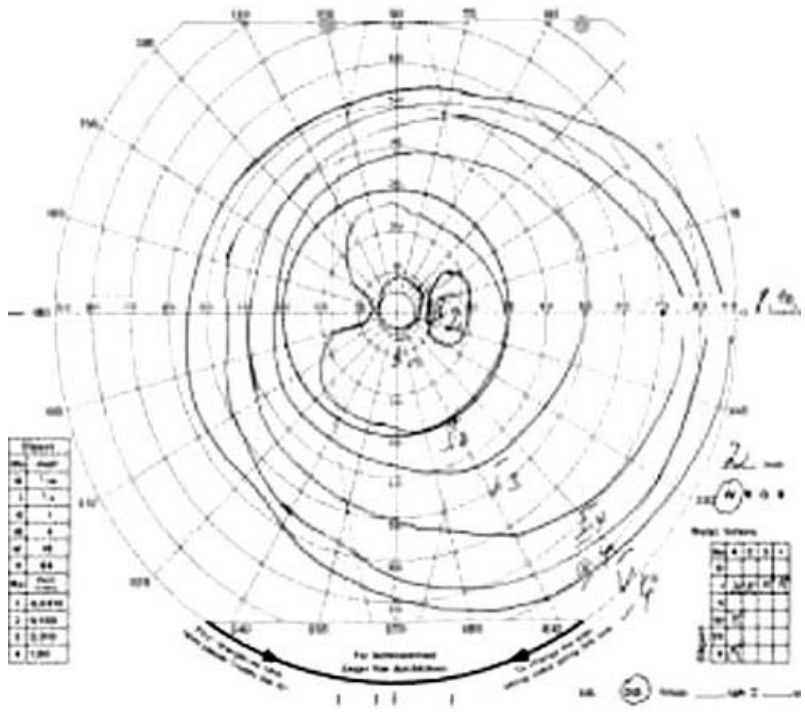

Figure 3 Patient 24. Operated without ICG. Loss of fixation, paracentral nasal defect.

The histological examination of peeled membranes showed cellular elements leading to the hypothesis of ICG causing retinal damage by an alteration of the vitreoretinal interface, ${ }^{19}$ whereas another histological study revealed similar cellular elements on membranes that were peeled without the aid of ICG. ${ }^{22}$ A possible photosensitising effect after intraoperative illumination of the stained retina with light of wavelengths between 380 and $760 \mathrm{~nm}$ as emitted by commonly used vitrectomy light pipes leading to

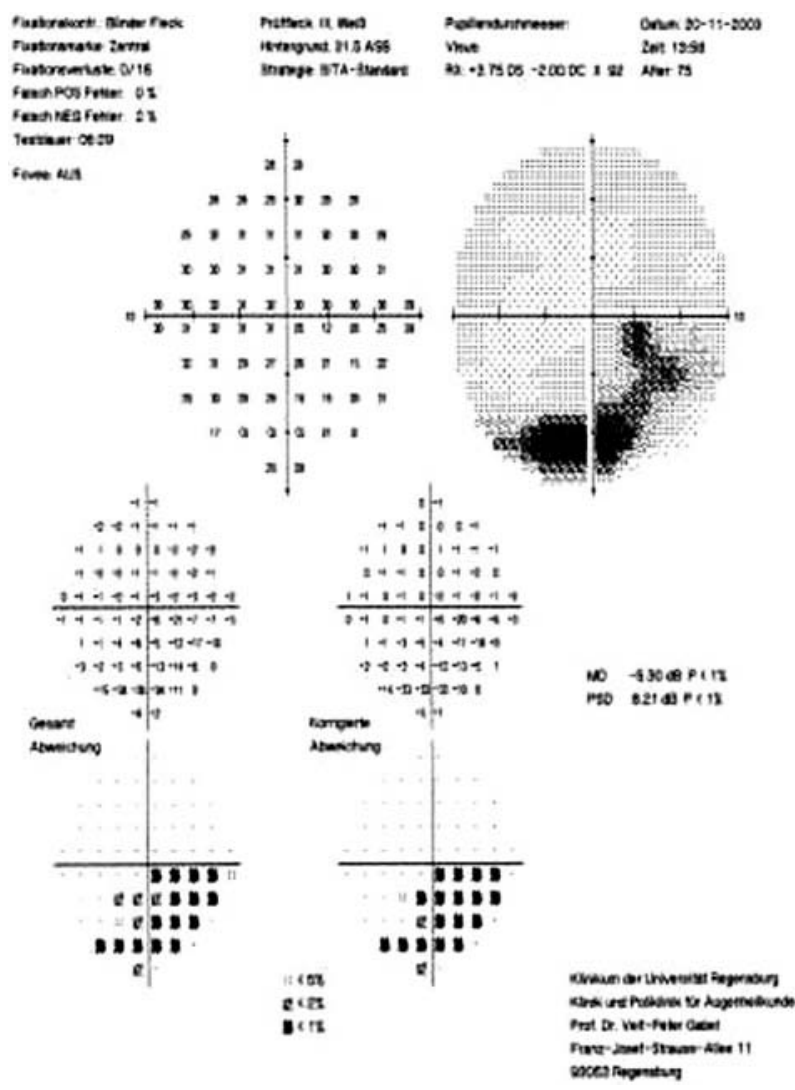

Figure 4 Patient 30. Operated without ICG. Defect only in HFA 10. 


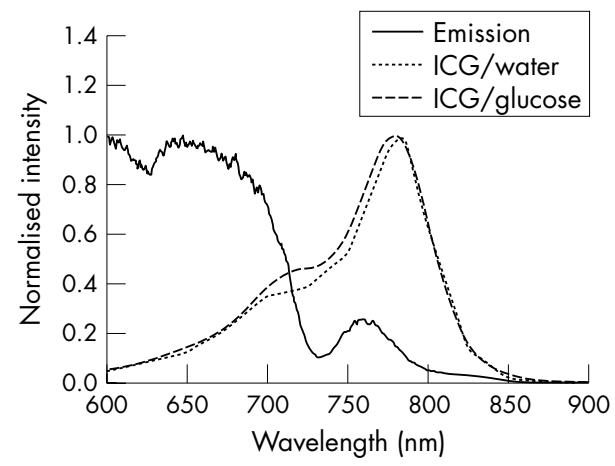

Figure 5 The normalised emission spectrum (solid line) of the light source (Xenophot 64634HLXEFR, 15V, 150W, Osram, Germany) and the normalised absorption spectrum of ICG in water (dotted line) and in glucose 5\% (broken line).

morphological damage of the inner retina has been reported. ${ }^{23}$ This finding was not confirmed by other investigations. ${ }^{25}{ }^{26} \mathrm{~A}$ possible cytotoxic effect of ICG has been investigated in experimental studies. Enaida et al found direct dose dependent functional and morphological damage of the retina after intravitreous application of ICG in an in vivo rat model. ${ }^{27}$ The doses used in this study were within or below the range $(0.05-5.0 \mathrm{mg} /$ eye $)$ of clinical application but in contrast with clinical practice, where the ICG solution is removed within seconds up to 1 minute, the authors left the ICG in the eye for 10 days before examination of the exposed retinas. ${ }^{27}$ The clinical relevance of the results is therefore questionable.

We found visual field defects in three patients operated without ICG versus one patient operated with ICG. It is a limitation of the present retrospective analysis that we cannot exclude the presence of the described asymptomatic visual field defects before surgery. However, the results do not show a correlation between the incidence of visual field defects and the use of ICG. Visual field defect after vitrectomy is a well known postoperative complication which has been attributed to a dehydration injury of the retina during fluidair exchange. ${ }^{28-30}$ This mechanism is unlikely to have had a role in our patients since we did not perform a fluid-air exchange during surgery. Visual field defect after vitrectomy has also been attributed to traction during cortical vitreous peeling, ${ }^{31}$ to intraoperative fluctuations of intraocular pressure, to optic nerve damage from retrobulbar injection, or to direct intraoperative mechanical trauma to the optic nerve. ${ }^{32}$ Kim et al reported a visual field defect after uneventful surgical removal of epiretinal membrane. Electron microscopy of the removed membrane showed adhesion between the epiretinal membrane and axons of the nerve fibre layer in an area of internal limiting lamina defect. ${ }^{33}$

We cannot conclude from our data which mechanism caused the visual defects in our patients but our data do not support the hypothesis of ICG as a likely cause. As cited above, there is principal experimental evidence of a direct dose dependent cytotoxic effect of ICG ${ }^{27}$ Also, phototoxicity of ICG irradiated with a diode laser emitting light at a wavelength of $805 \mathrm{~nm}$ has been studied in vitro. As the result, the photodynamic effect of ICG was related to the amount of ICG and the energy density of the illumination. ${ }^{34} 35$ The comparison of the emission spectrum of the light source used in the present study and the absorption spectrum of ICG show an overlap (fig 5) and a phototoxic effect can therefore not be excluded. However, the studies mentioned ${ }^{34}{ }^{35}$ do not necessarily reflect the actual intraoperative situation in macular surgery. 
Table 3 Statistical analysis of results comparing parameters before and after surgery within the patient group operated with ICG

\begin{tabular}{lll}
\hline With ICG $(\mathbf{n}=\mathbf{2 0 )}$ & BCVA (logMAR) & Macular retinal thickness ( $\boldsymbol{m m}$, OCT) \\
\hline Preoperative & $0.48(0.2)$ & $427(143)$ \\
Postoperative & $0.28(0.2)$ & $294(77)$ \\
Statistical analysis & $p=0.003$ & $p=0.001$ \\
& Wilcoxon test for paired samples & Wilcoxon test for paired samples \\
\hline \multicolumn{2}{l}{ All values given as mean (SD). } & \\
\hline
\end{tabular}

Table 4 Statistical analysis of results comparing parameters before and after surgery within the patient group operated without ICG

\begin{tabular}{lll}
\hline Without ICG $(\mathbf{n}=19)$ & BCVA (logMAR) & Macular retinal thickness ( $\mu \mathrm{m}, \mathrm{OCT})$ \\
\hline Preoperative & $0.49(0.18)$ & $415(114)$ \\
Postoperative & $0.27(0.18)$ & $347(85)$ \\
Statistical analysis & $p=0.003$ & $p=0.011$ \\
& Wilcoxon test for paired samples & Wilcoxon test for paired samples \\
\hline \multicolumn{2}{l}{ All values given as mean (SD). }
\end{tabular}

The absorption spectrum of ICG was very similar when dissolved in water or glucose 5\% (fig 5). This is in contrast with Haritoglou et al who compared glucose $5 \%$ and balanced salt solution as solvents using lower concentrations of ICG than those used in the present study. They reported a variation in absorption depending on the solvent medium, with a shift towards higher wavelengths when glucose 5\% was used as a solvent compared to balanced salt solution. ${ }^{36}$

In principle, retinal toxicity of ICG in macular surgery can be expected to depend on the concentration of the ICG solution, the osmolarity of the solution, ${ }^{37}$ the length of time before ICG is removed from the eye, ${ }^{27}$ whether ICG is injected into an air filled or a fluid filled eye (with less toxicity in fluid filled eyes), ${ }^{38}$ and the emission spectrum and the energy density of the illumination (fig 5). ${ }^{34}{ }^{35}$ However, the question of a specific toxicity threshold of the retina for ICG remains unanswered. Our results suggest that ICG assisted macular surgery for IEM in the setting described in the present study did not cause retinal toxicity detectable with the diagnostic tests applied. Less favourable results in visual acuity and visual field defects in some other studies may have been caused by a higher ICG concentration ${ }^{20}{ }^{21}$ and/or hypoosmolarity of the solvent. ${ }^{819202136}$ A recent experimental study showed retinal toxicity after subretinal but not after epiretinal application of ICG in rabbits. ${ }^{39}$ Therefore, retinal toxicity may also depend on the type of macular surgery, possibly because the full thickness retinal hole allows direct contact of ICG with deeper retinal layers and the RPE in macular hole surgery ${ }^{19}{ }^{20}$ but not in IEM surgery.

In summary, the results of the present study add relevant clinical data to the current controversial debate on the safety of ICG. ICG and other dyes should be used cautiously. Since ICG staining did not lead to better functional results in the present study and adverse effects can not yet be fully excluded, we recommend its use in epiretinal membrane peeling only when the membrane can otherwise not be safely removed. The functional and morphological results of the present study suggest that removal of epiretinal tissue with or without assistance of ICG improves visual function and reduces macular oedema in most patients.

The influence of enhanced cataract formation following vitrectomy must be taken into consideration in the evaluation of the functional outcome of our patients. Most of our patients whose visual acuity did not significantly improve were operated with surgical membrane peeling alone.
Therefore, it seems reasonable to recommend concomitant lens surgery in all patients older than 60 years.

\section{ACKNOWLEDGEMENT}

Indocyanine green was a kind gift from Pulsion Medical Systems AG, Munich, Germany.

\section{Authors' affiliations}

J Hillenkamp, P Saikia, F Gora, H G Sachs, C P Lohmann, V-P Gabel, Department of Ophthalmology, University of Regensburg, Germany J Roider, Department of Ophthalmology, Universitätsklinikum SchleswigHolstein, Campus Kiel, Kiel, Germany

W Bäumler, Department of Dermatology, University of Regensburg, Germany

Competing interests: None of the authors has a financial interest in indocyanine green.

Ethical approval: Approval was obtained from the ethics committee of the University of Regensburg.

Presented in part at the Annual Meeting of the Association for Research in Vision and Ophthalmology (ARVO), Fort Lauderdale, FL, USA, 25-29 April 2004.

\section{REFERENCES}

1 Mitchell P, Smith W, Chey T, et al. Prevalence and association of epiretinal membranes. The Blue Mountains Eye Study. Austral Ophthalmol 1997:104:1033-40

2 Tanikawa A, Horiguchi $M$, Kondo $M$, et al. Abnormal focal macular electroretinograms in eyes with idiopathic epimacular membrane. Am J Ophthalmol 1999;127:559-64.

3 Miyake Y, Miyake K, Shiroyama N. Classification of aphakic cystoid macular edema with focal macular electroretinograms. Am J Ophthalmol 1993; 1 16:576-83.

4 Wilkins JR, Puliafito CA, Hee MR, et al. Characterisation of epiretinal membranes using optical coherence tomography. Ophthalmology 1996; 103:2142-51.

5 Massin P, Allouch C, Haouchine B, et al. Optical coherence tomography of idiopathic macular epiretinal membranes before and after surgery. Am J Ophthalmol 2000;130:732-9.

6 Azzolini C, Patelli F, Codenotti M, et al. Optical coherence tomography in idiopathic epiretinal macular membrane surgery. Eur J Ophthalmol 1999;9:206-11.

7 Niwa T, Terasaki H, Kondo M, et al. Function and morphology of macula before and after removal of idiopathic epiretinal membrane. Invest Ophthalmol Vis Sci 2003;44:1652-6.

8 Haritoglou C, Gandorfer A, Gass CA, et al. The effect of indocyanine-green on functional outcome of macular pucker surgery. Am J Ophthalmol 2003; 135:328-37.

9 Sorcinelli R. Surgical management of epiretinal membrane with indocyaninegreen-assisted peeling. Ophthalmologica 2003;217:107-10.

10 Stalmans P, Parys-Vanginderdeuren R, De Vos R, et al. ICG staining of the inner limiting membrane facilitates its removal during surgery for macular holes and puckers. Bull Soc Belge Ophthalmol 2001;281:21-6. 
11 Kadonosono $\mathrm{K}$, Itoh $\mathrm{N}$, Uchio $\mathrm{E}$, et al. Staining of internal limiting membrane in macular hole surgery. Arch Ophthalmol 2000;118:1116-18.

12 Da Mata AP, Burk SE, Riemann CD, et al. Indocyanine- green-assisted peeling of the retinal internal limiting membrane during vitrectomy surgery for macular hole repair. Ophthalmology 2001;108:1187-92.

13 Kwok AK, Li WW, Pang CP, et al. Indocyanine green staining and removal of internal limiting membrane in macular hole surgery: histology and outcome. Am J Ophthalmol 2001;132:178-83.

14 Kampik A, Green WR, Michels RG, et al. Ultrastructural features of progressive idiopathic epiretinal membrane removed by vitreous surgery. Am J Ophthalmol 1980;90:797-809.

15 Kampik A, Kenyon KR, Michels RG, et al. Epiretinal and vitreous membranes. Comparative study of 56 cases. Arch Ophthalmol 1981;99:1445-54.

16 Weinberger AW, Schlossmacher B, Dahlke C, et al. Indocyanine-greenassisted internal limiting membrane peeling in macular hole surgery: a followup study. Graefes Arch Clin Exp Ophthalmol 2002;240:913-17.

17 Wolf S, Reichel MB, Wiedemann P, et al. Clinical findings in macular hole surgery with indocyanine-green assisted peeling of the internal limiting membrane. Graefes Arch Clin Exp Ophthalmol 2003;241:589-92.

18 Kwok AK, Lai TY, Man-Chan W, et al. Indocyanine green assisted retinal internal limiting membrane removal in stage 3 or 4 macular hole surgery. Br J Ophthalmol 2003;87:71-4.

19 Haritoglou C, Gandorfer A, Gass CA, et al. Indocyanine green-assisted peeling of the internal limiting membrane in macular hole surgery affects visual outcome: a clinicopathologic correlation. Am J Ophthalmol 2002;134:836-41.

20 Ando F, Sasano K, Ohba N, et al. Anatomic and visual outcomes after indocyanine green-assisted peeling of the retinal internal limiting membrane in idiopathic macular hole surgery. Am J Ophthalmol 2004;137:609-14.

21 Uemura A, Kanda S, Sakamoto Y, et al. Visual field defects after uneventful vitrectomy for epiretinal membrane with indocyanine green-assisted internal limiting membrane peeling. Am J Ophthalmol 2003;136:252-7.

22 Eckardt C, Eckardt U, Groos S, et al. Removal of the internal limiting membrane in macular holes. Clinical and morphological findings [German] Ophthalmologe 1997;94:545-51

23 Gandorfer A, Haritoglou C, Gandorfer A, et al. Retinal damage from ICG in experimental macular surgery. Invest Ophthalmol Vis Sci 2003;44:316-23.

24 Haritoglou C, Gandorfer A, Gass CA, et al. Histology of the vitreoretinal interface after staining of the internal limiting membrane using glucose $5 \%$ diluted indocyanine and infracyanine green. Am J Ophthalmol $2004 ; 137 \cdot 345-8$.
25 Grisanti S, Szurman P, Gelisken F, et al. Histological findings in experimental macular surgery with Indocyanine green. Invest Ophthalmol Vis Sci 2004;45:282-6.

26 Kadonosono K, Takeuchi, Yabuki K, et al. Absorption of short wavelengths of endoillumination in indocyanine green solution: implications for internal limiting membrane removal. Graefes Arch Clin Exp Ophthalmol 2003;241:284-6

27 Enaida H, Sakamoto T, Hisatomi T, et al. Morphological and functional damage of the retina caused by intravitreous indocyanine green in rat eyes. Graefes Arch Clin Exp Ophthalmol 2002;240:209-13.

28 Welch JC. Dehydration injury as a possible cause of visual field defect after pars plana vitrectomy for macular hole. Am J Ophthalmol 1997;124:698-9.

29 Ohij M, Nao-I N, Saito Y, et al. Prevention of visual field defect after macular hole surgery by passing air used for fluid-air exchange through water. Am J Ophthalmol 1999; 127:62-6.

30 Hasumura T, Yonemura N, Hirata A, et al. Retinal damage by air infusion during vitrectomy in rabbit eyes. Invest Ophthalmol Vis Sci 2000;41:4300-4

31 Ezra E, Arden GB, Riordan-Eva $\mathrm{P}$, et al. Visual field loss following vitrectomy for stage 2 and 3 macular holes. Br J Ophthalmol 1996:80:519-25.

32 Kerrison JB, Haller JA, Elman $M$, et al. Visual field loss following vitreous surgery. Arch Ophthalmol 1996;114:564-9.

$33 \mathrm{Kim}$ CY, Lee JH, Lee SJ, et al. Visual field defect caused by nerve fiber laye damage associated with an internal limiting lamina defect after uneventful epiretinal membrane surgery. Am J Ophthalmol 2002;133:569-71.

34 Bäumler W, Abels C, Karrer S, et al. Photo-oxidative killing of human colonic cancer cells using indocyanine green and infrared light. Br J Cancer 1999:80:360-3.

35 Fickweiler S, Szeimies RM, Bäumler W, et al. Indocyanine green: intracellular uptake and phototherapeutic effects in vitro. J Photochem Photobiol 1997;38:178-83.

36 Haritoglou C, Gandorfer A, Schaumberger M, et al. Light-absorbing properties and osmolarity of indocyanine-green depending on concentration and solvent medium. Invest Ophthalmol Vis Sci 2003:44:2722-9.

37 Stalmans $\mathbf{P}$, Van Aken EH, Veckeneer $M$, et al. Toxic effect of indocyanine green on retinal pigment epithelium related to osmotic effects of the solvent. Am J Ophthalmol 2002;134:282-5.

38 Czajka MP, McCuen BW, C-ummings TJ, et al. Effects of indocyanine green on the retina and retinal pigment epithelium in a porcine model of retinal hole. Retina 2004;24:275-82

39 Maia M, Kellner L, de Juan E Jr, et al. Effects of indocyanine green injection on the retinal surface and into the subretinal space in rabbits. Retina 2004;24:80-91. 